\title{
A Unique Clinical Presentation of Subclavian Steal Syndrome Provoked by Walking in a Patient with a Patent Axillo-Femoral Bypass
}

\author{
Nikolaos Kontopodis ${ }^{1}$, Nikolaos Galanakis ${ }^{2}$, Stavros Charalambous ${ }^{2}$, Elias Kehagias ${ }^{2}$, \\ Dimitrios Tsetis ${ }^{2}$, and Christos V. loannou ${ }^{1}$ \\ 'Vascular Surgery Unit, Department of Vascular and Cardiothoracic Surgery, ${ }^{2}$ Interventional Radiology Unit, \\ Department of Medical lmaging, University of Crete Medical School, Heraklion, Greece
}

An 80-year-old compromised man had undergone a unilateral left axillo-femoral and below-knee femoral-popliteal bypass 18 months earlier, owing to chronic limb-threatening ischemia with a postoperative ankle-brachial index $(\mathrm{ABI})$ of 0.9 . He reported no significant symptoms from the revascularized limb and experienced no ischemic ulcers or rest pain. He was able to walk enough to complete his activities

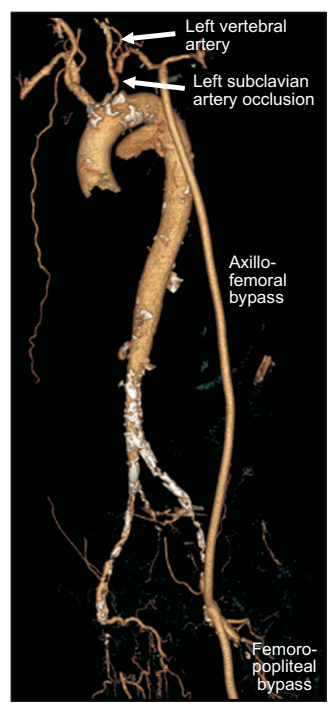

Fig. 1. Three-dimensional computed tomography angiography reconstruction showed a short occlusion of the proximal left subclavian artery and the patent axillo-femoral bypass graft. of daily living. The patient presented to our department with atypical symptoms of dizziness after walking a standard distance of $100 \mathrm{~m}$; this feeling subsided after a short time of rest. He reported that symptoms had been persisting for 2 weeks. He did not experience claudication, and his $\mathrm{ABl}$ was 0.65 . Color duplex ultrasonography revealed patent axillo-femoral and femoral-popliteal bypass grafts with a reduced peak systolic velocity of $50 \mathrm{~cm} / \mathrm{s}$. Color duplex ultrasonography of the carotid and vertebral arteries showed

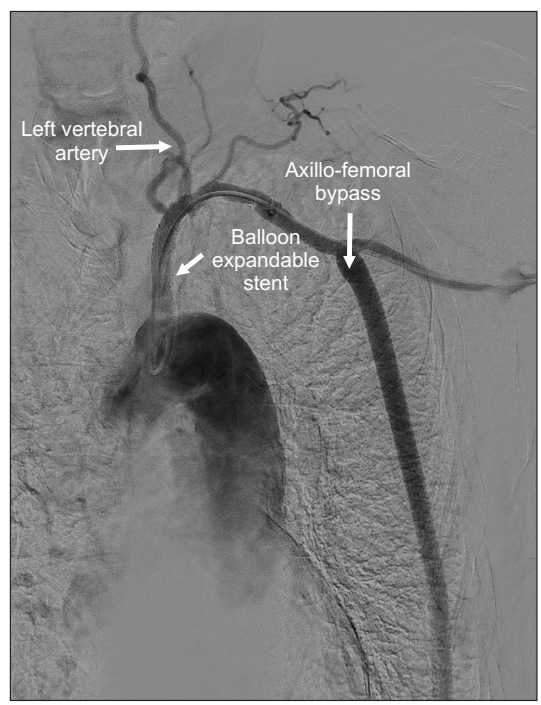

Fig. 2. Subclavian artery flow was restored using a balloonexpandable stent.

Received August 9, 2021, Revised August 30, 2021, Accepted September 12, 2021, Published online November 18, 2021 Corresponding author: Nikolaos Kontopodis, Vascular Surgery Unit, Department of Cardiothoracic and Vascular Surgery, University Hospital of Heraklion, University of Crete Medical School, PO-Box 1352, 71110, Heraklion, Greece

Tel: 30-2810-392-393, Fax: 30-2810-375-365, E-mail: kontopodisn@yahoo.gr, https://orcid.org/0000-0002-6792-5003

Copyright (c) 2021 The Korean Society for Vascular Surgery

This is an Open Access article distributed under the terms of the Creative Commons Attribution Non-Commercial License (http://creativecommons.org/licenses/by-nc/4.0) which permits unrestricted non-commercial use, distribution, and reproduction in any medium, provided the original work is properly cited.

Cite this article; Vasc Specialist Int 2021. https://doi.org/10.5758/vsi.210058 
complete flow reversal in the left vertebral artery. Computed tomography angiography revealed a short occlusion of the left subclavian artery, proximal to the origin of the vertebral artery (Fig. 1). Based on the clinical presentation and imaging examinations, subclavian steal syndrome from the lower limb during exercise was hypothesized to be the cause of this patient's symptoms [1,2]. Although both open surgical reconstruction and endovascular recanalization were feasible, angioplasty and stenting were recommended as most likely to provide improved symptom-free survival and freedom from re-intervention [3-5]. The patient, there- fore, underwent percutaneous recanalization of the lesion via a brachial approach, using a balloon expandable stent measuring $8 \mathrm{~mm} \times 37 \mathrm{~mm}$ (Visi-Pro Peripheral Stent System; Medtronic, Dublin, Ireland) (Fig. 2). After the procedure, he reported complete resolution of symptoms, and repeat color duplex ultrasonography revealed restoration of the antegrade blood flow in the ipsilateral vertebral artery. This report describes a unique clinical presentation of subclavian steal syndrome triggered by walking; this is possible in case of a stenosed subclavian artery serving as inflow for a peripheral arterial bypass.

\section{REFERENCES}

1) Kargiotis O, Siahos S, Safouris A, Feleskouras A, Magoufis G, Tsivgoulis G. Subclavian steal syndrome with or without arterial stenosis: a review. J Neuroimaging 2016;26:473-480.

2) Labropoulos N, Nandivada P, Bekelis K. Prevalence and impact of the subclavian steal syndrome. Ann Surg 2010;252:166-170.

3) Nam WS, Won YS. Carotid-axillary bypass for subclavian atery occlusion with gaining access through the axillary fossa: report of one case. J Korean Soc Vasc Surg 2010;26:128-131.

4) Do BS, Suh BY, Kwun KB. Axillo-axillary artery bypass graft for the treatment of subclavian steal syndrome: experience of 3 cases. Korean J Vasc Endovasc Surg 1991;7:36-41.

5) Byrne C, Tawfick W, Hynes N, Sultan S. Ten-year experience in subclavian revascularisation. A parallel comparative observational study. Vascular 2016;24:378-382. 